\title{
Dealing with activity in RV planet searches
}

\author{
Isabelle Boisse \\ Aix Marseille Université, CNRS, LAM (Laboratoire d'Astrophysique de Marseille) UMR 7326, \\ 13388, Marseille, France - isabelle.boisse@lam.fr
}

\begin{abstract}
Precise radial velocity measurements of a star allow to search for planets. But this method has to face with irregularly time series. Stellar variabilities: pulsation, granulation, stellar activity on a short and long timescale, also modify the measure of the radial velocities. There is indeed a growing literature of controversies on how a signal is interpreted as a planet or due to stellar activity. I present how the star variations change the measured RVs, which techniques and indices are used by several teams to disentangle activity and planets, and the future options that are being studied.
\end{abstract}

Keywords. Stellar activity, Spectroscopy,

\section{Introduction}

I focus this short review on the impact of stellar activity on radial velocity (RV) measurements. I omit most of the work done on its impact on planetary parameters from transit observations, and do not attempt an exhaustive review of the literature.

Precise RVs are derived from the measure of the Doppler shift of thousands of spectral lines. Dwarfs stars of spectral type F, G and K are the core of RV planet searches with several thousand of lines available in the visible wavelength range. Contrary to a Doppler effect du to an orbiting companion that shifts the line, stellar variability affects the line shape. Different timescales exist:

(a) pulsations induce variability of 0.1 to $4 \mathrm{~m} / \mathrm{s}$ on a few $\mathrm{min}$ ( $5 \mathrm{~min}$ for the Sun),

(b) flares have a signature of few $\mathrm{m} / \mathrm{s}$ that least hours,

(c) granulation impacts RV on 0.1 to $2 \mathrm{~m} / \mathrm{s}$ on hours,

(d) stellar activity, i.e. the rotation of spots and plages with the line of sight, leads to $\mathrm{m} / \mathrm{s}$ to $\mathrm{km} / \mathrm{s}$ variability with periods of days to weeks, and

(e) the long term variability, often related to magnetic cycle have a years basis with amplitudes of $\mathrm{m} / \mathrm{s}$ to $20 \mathrm{~m} / \mathrm{s}$.

These numbers are indicative and are not always well constrained. They are moreover dependent, at least, on the spectral type.

With the increase precision of RV spectrographs, the literature now has increasing controversies on the data analysis of RV surveys, depending on the way the data are analysed and how researchers take into account the stellar variability. Periodicities found in the RV could be interpreted as due to the star or to planets. Examples include CoRoT-7 (Haywood et al. 2014), HD 41248 (Santos et al. 2014, Jenkins \& Tuomi 2014), on GJ 581 (Robertson et al. 2015a, Anglada-Escude \& Tuomi 2015), and GJ 191 (Anglada-Escude et al. 2015, Robertson et al. 2015b).

\section{A clever observational strategy}

When the frequency of the noise (here the stellar variability) is different from the periodic one of the planet that we search, the noise should be averaged. For that, the 
way the measurements are treated is very important. Pulsation is averaged thanks to a 15 min exposure. Several measurements per night (2 or 3 depending on the spectral type) allow the average of the granulation noise. For a planet with a year-period, the stellar activity should be averaged thanks to several measurements per rotational period (Dumusque et al. 2011).

On the other hand, to analyse the RV amplitude induced by CoRoT-7b, Hatzes et al. (2011) proposed to analyse the data without modelling the activity signal. Even if the star is strongly active, since the planet has a period of 0.85 day, one can fit the planet periodic signal considering a free offset between each night. Since the rotational period of the star is $\sim 20$ days, one can consider that the stellar activity does not change much during one night.

A careful selection of the targets and a clever observational program is the first strategy of RV surveys to disentangle activity and orbiting companions.

\section{Observational diagnostics}

Since RV observations are coming from high-resolution spectra, several diagnostics are available to monitor the degree of activity of the star: line bisector variations (e.g. Vspan, BIS), active lines (CaII H\&K, H $\alpha$, HeI, NaI), and also simultaneous observations can be done in photometry, polarimetry or spectroscopy in the near infrared.

There was few cases of simultaneous high resolution spectroscopy and high precision photometry observations. HD189733 was monitored with SOHIE@OHP and the satellite MOST. It shows that the star is dominated by dark spots (Boisse et al. 2009, Lanza et al. 2011, Aigrain et al. 2012, Dumusque et al. 2014). The observations of CoRoT-7 with CoRoT and HARPS@LaSilla allow to refine the number and the masses of the planets in the system (Haywood et al. 2014, Barros et al. 2014). Spectroscopic observations of stars simultaneously observed by K2 are under study. These observations will help to understand how degenerate is the photometric signal and how it could be physically related to the spectroscopic one, but also its dependance on the stellar parameters.

There are several programs under analysis of simultaneous spectroscopic and polarimetric observations (Hébrard et al., subm.). The idea is to observe how the large scale magnetic field structure could help to understand the RV. The programs focus on different targets: on $\mathrm{M}$ dwarfs in the preparation of the SPIRou instrument (Moutou et al. 2015), on G and K dwarfs, and on young stars, cTTs and wTTs stars.

Soon, a new generation of nIR spectrographs will be on the sky (CARMENES, SPIRou, HPF, IRD). The impact of a dark spot is less important in the nIR than in the visible (e.g. Figuiera et al. 2010) and allow to disentangle between the signature of a planet and stellar activity.

\section{Simulation tools}

Several tools have been developed to simulate the RV and the photometry of dark spots and bright plages. Among them, SOAP 2.0 is a code available to the community $\dagger$ (Dumusque et al. 2014). It uses different mean spectral lines for spots, plages and quiet photosphere, that allows the modelling of convective blueshift and its inhibition in active regions. This is an evolving code that will be improved (differential rotation, evolution of the spot with time, simulated spectrum,...). The code StarsSim (Herrero et al., subm.) model the spectra with BT-Settl with Phoenix code. Spots and plages are simulated by

$\dagger$ http://www.astro.up.pt/resources/soap2/ 
stars with different temperature. An important challenge of thiese codes is to manage the number of free parameters when fitting the data.

\section{The Sun as a star}

The Sun is our closest star. The high spatial resolution available could be used to model the stellar activity impact (e.g. Meunier et al. 2010a,b, Lagrange et al. 2010, Borgniet et al. 2015, Marchwinski et al. 2015). Meunier \& Delfosse (2011) found that the correlation between the $\mathrm{H} \alpha$ and the CaII activity indices depends on the activity cycle.

The Sun has almost never been observed in the same way as the other stars are measured in RV, i.e. without the spatial resolution on the complete visible bandwidth. Recently observations were get with HARPS of Sunlight reflected on the VESTA asteroid (Haywood et al. subm.). We got a program accepted to observe reflected light on the Moon with HARPS (PI: P. Figueira), and a new instrument, the solar telescope is installed on HARPS-N@TNG. These observations could be compare to the high-spatial resolution observations obtained at the same time. This will allow to relate the RV, line width and active index variations to the appearance and disappearance of spots, plages and filaments.

\section{Conclusion}

In a few words to conclude: 1) the observational strategy has to be though anyway, 2) all the available diagnostics should be scrutinised, 3) we should be aware that the diagnostics may behave differently depending on spectral type, 4) in the modelling of observations, the red noise should be implemented.

\section{References}

Aigrain, S., Pont, F., \& Zucker, S. 2012, MNRAS, 419, 3147

Anglada-Escud, G., Tuomi, M., Arriagada, P. et al. 2015, arXiv: 1506.09072

Barros, S., Almenara, J., Deleuil, M. et al. 2014, A\&A, 569, 74

Boisse, I., Moutou, C., Vidal-Madjar, A. et al. 2009, A $\mathscr{E} A$, 495, 959

Boisse, I., Bouchy, F., Hébrard, G. et al. 2011, A\&A, 528, A4

Borgniet, S., \& Meunier, N. and Lagrange, A.-M. 2015, A\&A, 581, 133

Dumusque, X., Lovis, C., Ségransan, D. et al. 2011, A\& A, 535, 55D

Dumusque, X., Boisse, I., \& and Santos, N. C. 2014, ApJ, 796, 132

Figueira, P., Marmier, M., Bonfils, X. et al. 2010, A\&BA, 513, 8

Hatzes, A., Fridlund, M., Nachmani, G. et al. 2011, ApJ, 743, 75

Haywood, R., Collier Cameron, A., Queloz, D. et al. 2014, MNRAS, 443, 2517

Herrero, E., Ribas, I., Jordi, C. et al. 2015, hsa, 494

Jenkins, J. \& Tuomi, M. 2014, ApJ, 794, 10

Lagrange, A.-M., Desort, M., \& Meunier, N. 2010, A\& $A, 512,38$

Lanza et al. 2011, A\&SA, 533, A44

Marchwinski,R., Mahadevan, S., Robertson, P., Ramsey, L., \& Harder, J. 2015, ApJ, 798, 63

Meunier, N. et al. 2010a, A\&SA, 512, 39

Meunier, N. et al. 2010b, A\&SA, 512, 39

Meunier, N. \& Delfosse, X. 2011, A\& $A, 532,18$

Moutou, C., Boisse, I., Hébrard, G. et al. 2015, sf2a, arXiv:1510.01368

Robertson, P., Mahadevan, S., Endl, M., \& Roy, A. 2015a, Science, 347, 1080

Robertson, P., Roy, A., \& Mahadevan, S. 2015b, ApJ, 805, 22

Santos, N. C., Mortier, A., Faria, J. et al. 2014, A\&A, 566, 35 